\title{
O IMPACTO DAS PERDAS AUDITIVAS MÍNIMAS E LEVES E AÇÕES PARA O MANEJO EM SALA DE AULA
}

\section{THE IMPACT OF MINIMAL AND MILD HEARING LOSS, AND ACTIONS TO THE MANAGEMENT IN CLASSROOM}

\section{EL IMPACTO DE LAS PÉRDIDAS AUDITIVAS MÍNIMAS Y LEVES Y ACCIONES PARA EL MANEJO EN AULA}

\author{
Giovanna Castilho Davatz Lopes \\ Fonoaudióloga graduada pela USP- Bauru, Mestre e Doutoranda em Ciências pelo Programa \\ de Pós-Graduação Interunidades em Bioengenharia - EESC/FMRP/IQSC-USP. \\ Especializanda em Neuropsicopedagogia Clínica pela Censupeg. Atua como docente da \\ disciplina de Anatomia Humana nas FIJ-Faculdades Integradas de Jaú, desde 2011. \\ E-mail: giovannadavatz@usp.br.
}

\section{RESUMO}

Quando um escolar apresenta dificuldades de aprendizagem ou de alfabetização, qual será o problema? O método de ensino? A formação do professor? A resposta pode ser a existência de perda auditiva mínima ou leve, que acomete de $15 \%$ a $30 \%$ dos alunos e na maioria dos casos não é detectada. O presente artigo visa apresentar essa condição de saúde esclarecendo os agravos e possibilidades de manejo em sala de aula.

Palavras-chave: Perda Auditiva. Aprendizagem. Sala de aula.

\begin{abstract}
Whenever a student presents learning or literacy difficulties, which should be the problem? The teaching method? The teacher formation? The answer can be the existence of minimal or mild hearing loss, which occurs in $15 \%$ to $30 \%$ of the students, and in the most of cases is not detected. The present article aims to present this health condition, elucidating its grievances and the possibilities of management them in classroom.
\end{abstract}

Keywords: Hearing loss. Learning. Classroom.

\section{RESUMEN}

Cuando un alumno presenta dificultades de aprendizaje o de alfabetización, ¿Cuál será el problema? ¿El método de enseñanza? ¿La formación del profesor? La respuesta puede ser la existencia de pérdida auditiva mínima o leve, que afecta aproximadamente de $15 \%$ a $30 \%$ de los alumnos y en la mayoría de los casos no se detecta. El presente artículo tiene como objetivo presentar esta condición de salud aclarando los agravamientos y posibilidades de manejo en el aula.

Palabras clave: Pérdida auditiva. Aprendizaje. Aula. 


\section{INTRODUÇÃO}

A audição consiste na percepção e interpretação dos sons recebidos do meio. Este sentido depende da integridade de estruturas periféricas e centrais que no conjunto constituem o sistema auditivo: a orelha, que se divide em porção externa, média e interna; estruturas do tronco encefálico e o córtex auditivo, localizado no lobo temporal (ALBERTI, 2019).

Da orelha externa fazem parte o pavilhão auricular e meato acústico externo, com função de captar o som e conduzir para a orelha média. O pavilhão também exerce função de amplificação do sinal acústico. Da orelha média fazem parte o tímpano e a cadeia de ossículos que amplificam o som e o transformam em estímulo mecânico - movimento. O último ossículo movimenta um líquido presente na orelha interna, a perilinfa. Ao movimentar-se, esse líquido induz movimentos das células ciliadas, que são estruturas sensoriais localizadas dentro da cóclea. Nesse processo, o estímulo mecânico é modificado em estímulo neural e segue pelo nervo auditivo por estruturas do tronco encefálico até chegar ao córtex temporal, onde é interpretado. Da orelha interna também faz parte o sistema vestibular, relacionado ao equilíbrio (ALBERTI, 2019).

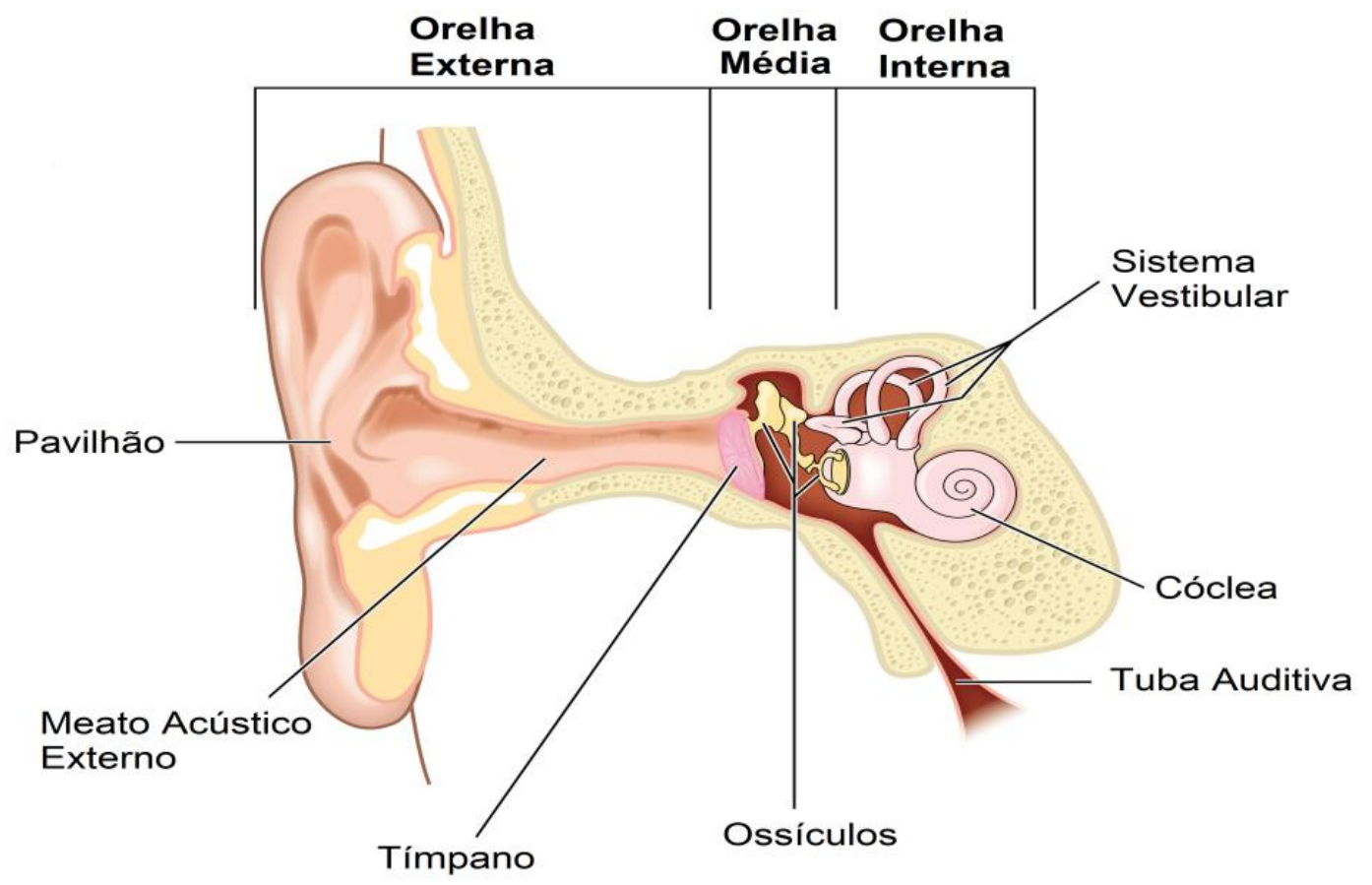

Figura 1: Anatomia da Orelha (Imagem adaptada Grath, 2019). 


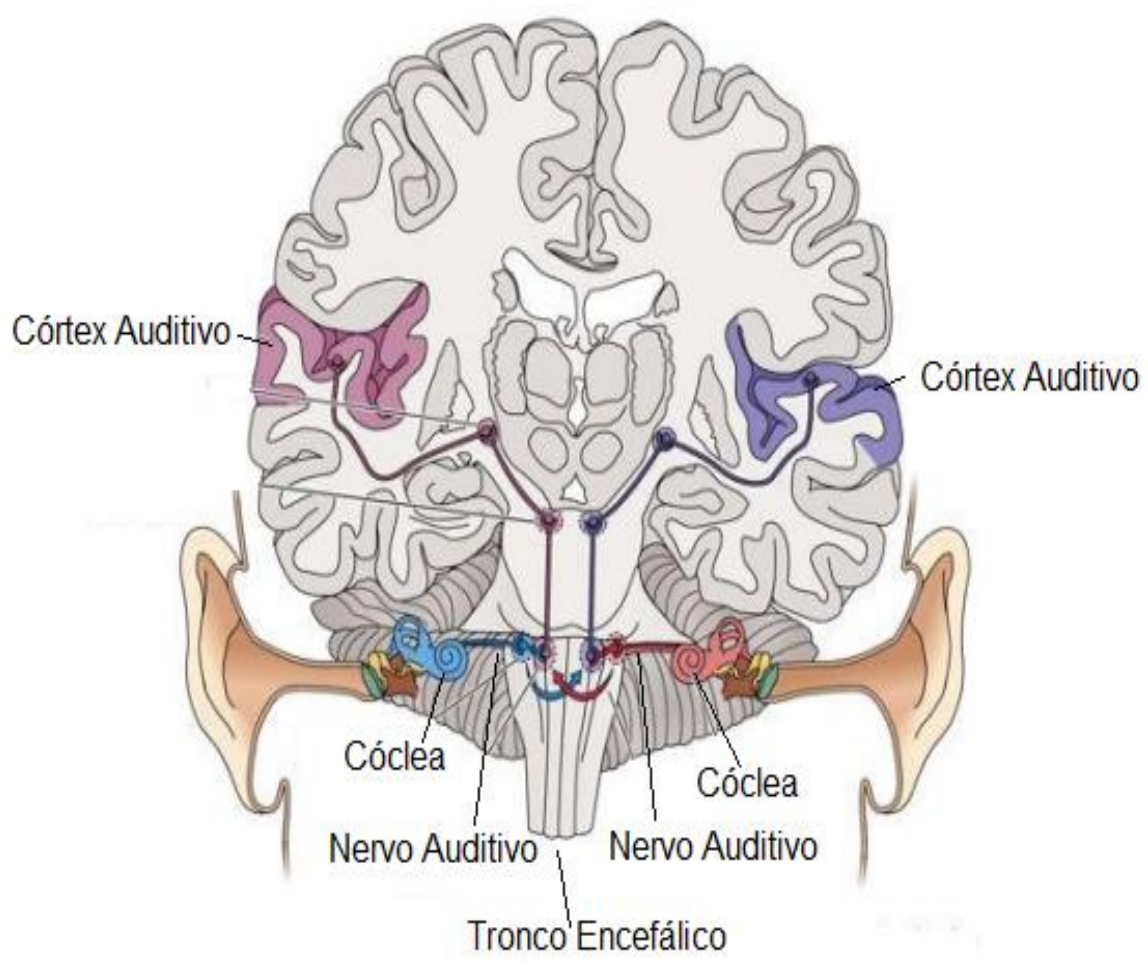

Figura 2: Áreas neurais da audição (Imagem adaptada de Sinauer, 2019).

A perda auditiva consiste em um conjunto heterogêneo de manifestações clínicas: quanto ao grau, há perdas leves, moderadas, severas e profundas; quanto ao tipo, há as condutivas, as sensoriais, as mistas e as neurais; quanto ao tempo de duração, há as temporárias e as permanentes (ZAHNERT, 2011).

Considera-se uma audição normal aquela em que o menor estímulo sonoro em que o indivíduo é capaz de captar é de até 15 decibéis $(\mathrm{dB})$. Há perda mínima quando a menor percepção auditiva ocorre para sons entre 16 e $20 \mathrm{~dB}$. Na perda leve o limiar auditivo está entre 25 e 40 dB. Na perda auditiva moderada a percepção auditiva ocorre quando o som se encontra entre 41 e $55 \mathrm{~dB}$. A perda moderadamente severa é quando a menor percepção do som se dá para 56 a $70 \mathrm{~dB}$. A perda severa corresponde a limiar auditivo entre 71 a $90 \mathrm{~dB}$. Na perda profunda a menor percepção sonora ocorre para sons a partir de 91 dB (WERFEL; HENDRICK, 2016).

Sobe o tipo de perda, descreve-se como condutiva aquela que ocorre nas porções externa ou média da orelha, prejudicando a condução do som até a orelha interna. A sensorioneural é aquela em que há morte de células ciliadas da cóclea, dificultando a percepção de alguns sons. As mistas são as que possuem componente condutivo associado ao sensorioneural. Nas neurais 
pode haver comprometimento do nervo auditivo ou de estruturas do sistema nervoso central (WHO, 2015).

Perdas auditivas permanentes são irreversíveis e podem ter como causa lesões neurais ou a morte de células ciliadas decorrente de exposição a ruídos ou músicas com intensidade elevada, ou pelo uso de medicamentos ototóxicos, por exemplo. Perdas temporárias são aquelas em que quando a causa é tratada, a sensibilidade auditiva é restaurada. Como causa dessas perdas temos a maior produção de cerúmen e uso de cotonetes, que o empurra para o fundo do meato, formando uma rolha; infecções como as otites médias e otite externa e presença de secreções em tuba auditiva decorrentes de resfriado (WHO, 2015).

Perdas severas e profundas congênitas tendem a ser detectadas precocemente e tratadas adequadamente. Estes casos são amplamente discutidos na educação, em decorrência da necessidade de utilização de amplificação sonora e muitas vezes da língua de sinais, além da realização de adaptações curriculares. Ocorre que estas perdas são raras, atingindo de 1 a 2 indivíduos a cada mil nascidos. Perdas mínimas e leves, no entanto, são frequentes e nem sempre são detectadas, uma vez em que a audição se encontra ligeiramente abaixo do padrão considerado normal. Crianças com esses graus de perda ouvem as palavras e falam, tendo dificuldades na percepção de alguns sons, o que faz com que pareçam apenas distraídas (WERFEL; HENDRICK, 2016).

São escassos os trabalhos em português que esclarecem sobre os impactos das perdas auditivas mínimas e leves em sala de aula ou no processo de alfabetização e as atividades que podem ser adotadas para minimizar os agravos. O presente artigo visa suprir essa necessidade, apresentando os achados presentes na literatura internacional com o objetivo de fornecer subsídios para a ação de professores e gestores escolares.

\title{
2 PERDAS AUDITIVAS MÍNIMAS E LEVES EM ESCOLARES
}

Mesmo 28 anos após o artigo de Tharpe e Bess (1991) que denuncia a falta de discussão e divulgação do manejo nos casos de perdas auditivas mínimas e leves em crianças, ainda pouco se fala sobre este aspecto na área da educação. É comum ouvirmos queixas quanto ao sistema educacional, diante de falhas do processo educativo nos anos iniciais, principalmente relacionadas às dificuldades de alfabetização. O quanto podemos acusar os métodos empregados ou a formação docente, quando não temos certeza nem sequer se os alunos

\author{
RELEDUC | ISE | v. 2 | n. 1 | fev. 2019
}


possuem vias sensoriais integras capazes de receber as informações fornecidas pelos professores?

Considerando a tendência do aumento do número de perdas auditivas com a idade, em decorrência de fatores como exposição ao ruído, sabe-se que muitos adultos possuem perdas auditivas mínimas ou leves. Entretanto, “adultos não apresentam prejuízos funcionais quando comparados a ouvintes normais" (THARPE, 2008). O grande impacto se dá em crianças, por estarem fase de desenvolvimento da linguagem oral e escrita.

É necessário identificar a perda auditiva das crianças antes de seu ingresso na escola (THARPE; BESS, 1999), entretanto há estimativas de que menos de 1\% dos escolares com esse quadro são identificados (KADERAVEK, PAKULSKI, 2002). Esta dificuldade de detecção dos acometidos se dá devido ao fato das triagens auditivas escolares serem feitas em uma intensidade sonora de $20 \mathrm{~dB}$, o que faz com que grande parte das crianças com mínimos níveis de perda, de 16 a 25 dB, não sejam identificadas (KADERAVEK,. PAKULSKI, 2002).

Além disso, alunos com a audição normal podem passar a apresentar alterações por vários fatores. As maiores causas de perdas auditivas nessa população $(88,9 \%)$ são temporárias e condutivas, estando relacionadas ao cerúmen e às otites (DAUD ET AL, 2010). Por isso, deve-se ter atenção com crianças que apresentem resfriados frequentes, infecções de garganta ou doenças respiratórias (KADERAVEK, PAKULSKI, 2002). Reforça-se também, a necessidade de cuidado e orientações aos pais, uma vez que estar exposto a ruídos em alta intensidade ou música amplificada durante momentos de lazer viabilizam o surgimento de perda de audição permanente nas crianças (THARPE; BESS, 1999), fator muito frequente com o uso de fones de ouvido em equipamentos como celulares e tablets.

Existem casos de crianças que nascem com audição normal e desenvolvem perda auditiva progressiva, ou seja, que se agrava dia após dia. Dentre os fatores causais se encontram questões de ordem genética, doenças durante o desenvolvimento gestacional como citomegalovírus, rubéola ou sífilis. Outros fatores de risco são a hiperbilirrubinemia, doenças autoimunes, Meniere, Meningite e a prematuridade (THARPE; BESS, 1999).

Perdas auditivas mínimas ou leves podem ser unilaterais, bilaterais ou em altas frequências (THARPE; BESS, 1999) e devido à sua diversidade etiológica, estudos epidemiológicos apontam que podem atingir de 15\% (WAKE, POULAKIS, 2004) a 30\% (KADERAVEK, PAKULSKI, 2002) dos escolares em alguma fase da vida acadêmica. 


\section{IMPACTOS NA LINGUAGEM E NO DESEMPENHO ACADÊMICO}

Perdas auditivas mínimas ou leves prejudicam a percepção auditiva dos fonemas surdos como /s/, /t/, /f/ ou /p/ (THARPE; BESS, 1999; NEW ZELAND GOVERNEMENT, 2014). Além disso, torna um sussurro ou fala muito suave inaudível mesmo em um ambiente silencioso (KADERAVEK, PAKULSKI, 2002). Assim, partes da fala do interlocutor parecem ausentes ou distorcidas, o que resulta em não entendimento de 10\%, quando há perda auditiva mínima e de até 50\% nas perdas de grau leve (ANDERSON E MATKIN, 2007).

Infantes com esses graus de perda de audição apresentam agravos no aprendizado da língua (KADERAVEK, PAKULSKI, 2002), com um ano ou mais de atraso no desenvolvimento da linguagem (YOSHINAGA-ITANO ET AL, 2008).

Nesses casos há tanto prejuízo de discriminação fonológica, consciência fonológica e memória fonológica quanto o apresentado em quadros de distúrbios específicos de linguagem.

Relacionando-se as informações, observa-se que a oscilação da percepção auditiva dos fonemas surdos (NEW ZELAND GOVERNEMENT, 2014) prejudica a formação e organização fonêmica a nível cognitivo, o que justificaria a defasagem da discriminação, memória e consciência fonológica descritos por Tharpe, (2008).

As habilidades fonológicas são parte do processo de alfabetização, uma vez que deve haver um adequado entendimento da organização dos fonemas com as possíveis posições que ocupam nas diferentes palavras para que posteriormente a criança faça a correta correlação com o grafema correspondente.

Essas crianças também possuem falhas de ordem articulatória, redução do vocabulário receptivo e expressivo; e dificuldade na sequencialização de palavras para se expressar, que se manifesta como má estruturação sintática e gramatical. Também apresentam desempenho reduzido em atividades de recontagem de estórias (THARPE; BESS, 1999; THARPE, 2007; THARPE, 2008).

Em avaliações objetivas, detectou-se defasagem em testes acadêmicos de desempenho verbal, de linguagem escrita (THARPE; BESS, 1999; THARPE, 2008) e de habilidades básicas de compreensão (YOSHINAGA-ITANO ET AL, 2008), o que em longo prazo pode ocasionar graves problemas de leitura (WAKE, POULAKIS, 2004). Não foi encontrado prejuízo em habilidades matemáticas (YOSHINAGA-ITANO ET AL, 2008).

Estudantes com perda auditiva mínima e leve mostram-se significativamente $(\mathrm{p}<0,001)$ associados ao baixo desempenho acadêmico (DAUD et al, 2010). Ressalta-se que em fase de

RELEDUC | ISE | v. 2 | n. 1 | fev. 2019 
alfabetização, de $50 \%$ a $80 \%$ das crianças com dificuldades no processo possuem esses graus de perdas auditivas, o que reforça os efeitos negativos da perda auditiva na estruturação fonêmica e seu impacto no aprendizado da escrita (WERFEL E HENDRICK, 2016).

O mau desempenho acadêmico gera danos de ordem emocional e comportamental, prejudicando a qualidade de vida das crianças e de suas famílias (DAVIS ET AL, 2002). Yoshinaga-Itano et al (2008) encontraram, nestes escolares, baixos escores em testes que envolvem comportamento, energia, apoio social e autoestima, e maiores comprometimentos em aspectos relacionados ao estresse, quando comparado a seus pares com audição normal.

\section{OBSERVANDO SINAIS EM SALA DE AULA}

Considerando-se a possibilidade da existência de indivíduos acometidos em todas as salas de aula, quais sinais apresentados pelos alunos podem levar o docente a percebê-los como pertencentes ao grupo de risco para perda auditiva mínima ou leve?

Em período pré-escolar é comum ouvir relatos dos pais de que não compreendem o que o filho diz (DAVIS ET AL, 2002), o que sinaliza possível dificuldade de percepção de sons da língua e consequentes falhas na reprodução dos mesmos.

Também se mostram como sinais de risco durante o desenvolvimento o ato de balbuciar quando se tem 1 ano de idade, ao invés de falar algumas palavras; aos dois anos compreender somente ordens simples; aos três anos ser incapaz de repetir frases com mais de duas palavras; aos quatro anos não conseguir estruturar o discurso a ponto de falar espontaneamente sobre o que acontece ou não compreender o que o interlocutor diz (CONAFE, 2010).

Crianças com dificuldades mesmo que mínimas da audição repetem muito a pergunta "O que?"; ao telefone nem sempre respondem a perguntas que lhes são feitas; ignoram sons ambientais de baixa intensidade como o barulho da chuva, do vento ou o canto de pássaros à distância (CONAFE, 2010).

Outras manifestações são confundir palavras semelhantes, utilizar frases muito simples para se comunicar, falar "muito alto", reclamar de "dor de ouvido" ou respirar pela boca (CONAFE, 2010).

$\mathrm{O}$ ato de mexer muito na orelha pode estar relacionado a quadros de infecções nesse órgão (KADERAVEK, PAKULSKI, 2002).

Crianças com esses graus de perda mostram dificuldades de escuta em ambientes com ruído, assim, ao invés de seguirem as instruções dadas pelo professor, tendem a copiar as ações

$$
\text { RELEDUC | ISE | v. } 2 \text { | n. } 1 \text { | fev. } 2019
$$


de colegas. Outra característica é o aluno aparentar "ouvir quando quer" ou "sonhar acordado". (ANDERSON E MATKIN, 2007).

Devido ao esforço para ouvir e compreender o professor, o aluno pode demonstrar forte cansaço, o que prejudica sua habilidade de atenção, a realização de anotações e a execução de tarefas em sala de aula (ANDERSON E MATKIN, 2007; THARPE, 2008). Alunos com leves prejuízos da audição também tendem a mostrar-se distraídos (KADERAVEK, PAKULSKI, 2002).

Outro sinal é o baixo desempenho acadêmico, que deve sempre ser observado (DAVIS ET AL, 2002), uma vez que grande parte das informações fornecidas verbalmente pelo professor não são percebidas e entendidas por esses alunos, interferindo no aprendizado global.

\title{
5 AÇÕES PARA O MANEJO EM SALA DE AULA
}

Analisando-se as sugestões de atividades presentes na literatura internacional para o manejo das perdas auditivas mínimas ou leves em ambiente escolar, nota-se que estas podem ser divididas em 1) ações centradas na criança; 2) mudanças atitudinais do professor, 3) ações de ordem educacional ou 4) modificações acústicas do ambiente.

\section{1) Ações centradas na criança:}

Todas as crianças que demonstram problemas de linguagem, no aprendizado ou na alfabetização devem ser encaminhadas para avaliação auditiva (WAKE, POULAKIS, 2004; WERFEL; HENDRICK, 2016).

Em casos de suspeitas ou de crianças já identificadas, sugere-se realizar atendimentos individuais para sanar as dificuldades acadêmicas.

Mostra-se como um fator de auxilio ao suporte e reabilitação, a colaboração de equipes multiprofissionais no ambiente escolar, uma vez que fonoaudiólogos, otorrinolaringologistas, pediatras, psicólogos e educadores especiais podem trazer muitas contribuições para a atuação do professor em sala de aula (WERFEL; HENDRICK, 2016).

Há crianças que poderão necessitar de adaptação de aparelho auditivo ou de sistema FM, equipamento que amplifica a voz do professor apenas para o aluno alvo, para melhorar a percepção e compreensão da voz do professor (KADERAVEK,. PAKULSKI, 2002; WERFEL; HENDRICK, 2016).

\author{
RELEDUC | ISE | v. 2 | n. 1 | fev. 2019
}


Pode se fazer necessária intervenção fonoaudiologia para tratamento de sintomas relacionados a dificuldades de articulação das palavras, além de auxilio no desenvolvimento da consciência fonológica, correção de falhas da correlação fonema-grafema e aprimoramento de habilidades gerais de comunicação oral. Profissionais da educação especialistas na área de leitura e escrita podem promover uma melhor estruturação frasal e gramatical, ampliação do vocabulário e remediação junto às habilidades de elaboração escrita e compreensão leitora.

Quanto mais precoces forem a detecção e intervenção nos casos de perdas auditivas mínimas e leves, maiores serão as possibilidades das crianças apresentarem resultados acadêmicos e de linguagem similares às com audição normal (WAKE, POULAKIS, 2004).

2) Mudanças atitudinais do professor:

Os professores devem estar bem treinados quanto a estratégias gerais de comunicação (THARPE, BESS, 1991). Por exemplo, manter o contato visual com a criança favorece sua atenção. O uso expressões faciais e gestos fornecem informações adicionais que enriquecem a mensagem (NEW ZELAND GOVERNEMENT, 2014). Outra sugestão é o uso de palavras a mais nas explicações, pois auxiliam no entendimento (KADERAVEK, PAKULSKI, 2002).

Considerando-se que as crianças com perda auditiva escutam melhor quando o interlocutor se encontra a até 1 metro de distância, mostra-se benéfico se aproximar de crianças que apresentem sinais de perda auditiva, enquanto se estiver falando, sanando dúvidas ou fazendo explicações de conceitos relevantes (NEW ZELAND GOVERNEMENT, 2014).

Para auxiliar na redução do ruído de fundo o professor pode ainda manter portas e janelas fechados (NEW ZELAND GOVERNEMENT, 2014).

3) Ações de ordem educacional:

\section{1) Intervenção para um adequado desenvolvimento da escrita:}

a) Nível fonético: Treinar a emissão dos sons da língua iniciando-se pelas vogais. A criança primeiramente deverá ouvir o som, depois tocar em região de mandíbula, boca, lábios e língua a fim de identificar a posição dos mesmos para sua emissão. Por exemplo, para a emissão do /a/ reforçar o fato da mandíbula estar abaixada e a língua, acoplada a ela. Após, deverá emitir o som sem a pista tátil (CONAFE, 2010).

b) Nível fonológico: Depois de a criança conhecer os fonemas com maneira adequada de emissão oral, por exemplo, o /p/, treinar a formação de sílabas com modelo, como o 
/pa/, e após, pedir para a criança inverter a vogal visando a formação de novas sílabas. Após, solicitar que forme palavras com essas sílabas, que podem ser reais ou inventadas como/papa/, /pepe/, /pipa/. Segue-se a esse trabalho relacionar a silaba com as formadas por outras consoantes como em /pato/, /pico/, /polo/. Depois dessa etapa, solicitar a construção de frases que contenham a palavra assinalada com o fonema alvo (CONAFE, 2010).

c) Relação fonema-grafema: Inicia-se o trabalho estimulando a criança a relacionar imagens conhecidas ao som, como por exemplo, uma vaca ao "muuuu". Cognitivamente, este tipo de relação antecede a relação de grafemas aos respectivos fonemas (CONAFE, 2010).

3.2) Intervenção para um adequado desenvolvimento da leitura: A sequência recomendável para treino de decodificação é iniciar com leitura de palavras monossílabas que possuam o fonema alvo, por exemplo, /pa/, seguindo-se por palavras dissílabas, como /pipa/, palavras trissílabas como /pipoca/, após, palavras polissílabas como /papagaio/ e por último as que possuam encontros consonantais como em /prato/ (CONAFE, 2010).

A cada etapa de progressão da leitura, apresentar diferentes orações que possuam as palavras alvo, para que sejam identificadas (CONAFE, 2010).

Deve-se reforçar a diferença entre as palavras e que em caso de troca ou omissão de sons ou letras, há tendência de formarmos outras palavras. Apresentam-se imagens dos objetos relacionados para favorecer a relação com o significado (CONAFE, 2010).

3.3 Intervenção para a compreensão leitora: Para atuação com os alunos com dificuldades auditivas orienta-se iniciar com perguntas curtas com vocabulário simples para favorecer sua compreensão. O uso de imagens e apoio auditivo são indicados como pistas adicionais ao texto (CONAFE, 2010).

No inicio, utilizar textos para interpretação literal. Solicitar que o aluno identifique o nome de personagens, local em que se passam os acontecimentos ou sequencias das ações. Depois se trabalha o nível inferencial, faz-se isso relacionando fatos do texto com o conhecimento prévio das crianças e encontrando ideias principais não explicitadas na estória. Para desenvolvimento do nível critico, apresentam-se conceitos em texto lido que devem ser aceitos ou rejeitados pelos alunos. O julgamento requerido pode ser se partes do texto podem ser reais ou se são fantasia; se são adequados e válidos para a resolução dos fatos apresentados, ou se são aceitáveis 11

RELEDUC | ISE | v. 2 | n. 1 | fev. 2019 
ou rejeitados dentro do sistema de valores da sociedade. O último é o nível criativo, em que a partir do texto a criança estrutura novos parágrafos, ou elaborar um diário de um dos personagens, ou ainda dramatizar a estória (CONAFE, 2010).

\section{4) Modificações acústicas do ambiente:}

Salas de aula são ambientes verbais-auditivos e por isso devem ter condições de escuta favoráveis (DAUD et al, 2010).

O ambiente acústico de uma sala de aula deve ter tempo de reverberação inferior a 0,5 segundo (THARPE, BESS, 1991; LEWIS, VALENTE, SALDING, 2016). Carpetes, cortinas ou persianas nas janelas ou uso de caixa de ovo em pontos da parede reduzem a reverberação do som (KADERAVEK, PAKULSKI, 2002).

Ruídos em sala de aula afetam pouco as crianças com audição normal, mas são altamente prejudiciais para casos de perda auditiva mínima ou leve (WAKE, POULAKIS, 2004). Para uma boa recepção auditiva de informações em sala de aula, o ruído ambiente deve ser inferior a $35 \mathrm{~dB}$. Valores superiores prejudicam a inteligibilidade de fala, a compreensão, as habilidades cognitivas e atividades que envolvam interação ou conversação (LEWIS, VALENTE, SALDING, 2016).

Para redução de ruído equipamentos como ar condicionado, ventiladores e lâmpadas devem estar adequadamente reparados. O uso de tampos de borracha nos pés das cadeiras ou a colocação de tapetes também alivia o ruído (KADERAVEK, PAKULSKI, 2002).

Recomenda-se, ainda, a amplificação da voz do professor em toda a ocasião (THARPE, BESS, 1991), uma vez que estando em $15 \mathrm{~dB}$ acima da intensidade do ruído não se mostra suficiente para a compreensão auditiva dos alunos acometidos (DAUD ET AL, 2010). A literatura aponta que a voz do professor deve estar entre 20 a $30 \mathrm{~dB}$ mais intensa que o ruído, além disso deve-se falar de forma clara, uma vez que a inteligibilidade da fala diminui quando se "fala alto" ou gritando (KADERAVEK, PAKULSKI, 2002).

\section{CONSIDERAÇOES FINAIS}

No contexto das perdas auditivas mínimas e leves, considerando que a maioria dos escolares que apresentam não é identificada e que devido ao cerúmen, otites e outras condições, muitas crianças com audição normal manifestarão perdas temporárias em algum período da 
vida acadêmica, mudanças atitudinais do professor ou ajustes acústicos na sala de aula, podem ser de grande valia para o aprendizado.

Com a abordagem utilizada no presente artigo, nota-se que aproximar saúde e educação não significa "patologizar" o aprendizado, mas compreender que as condições orgânicas do educando devem ser favoráveis para que este possa desenvolver ao máximo suas potencialidades.

Dentro da perspectiva de escola democrática e da inclusão, políticas que unam saúde e educação se mostram como uma alternativa tanto para detecção e intervenção precoces de diferentes condições, como para o surgimento e disseminação de novos conceitos, ações e estratégias que favoreçam o processo de ensino-aprendizagem.

Ambientes acessíveis para estudantes que tenham perdas auditivas mínimas ou leves devem ter tratamento acústico adequado, com baixo tempo de reverberação, redução do ruído de fundo e amplificação da voz do professor, aspectos que devem ser implementados visando a acessibilidade na educação.

\section{REFERÊNCIAS}

ALBERTI, P.W. The anatomy and physiology of the Ear and hearing: World Health Organization. Ocupational health. Noise 2. p. 53 a 62. Disponível em: https://www.who.int/occupational_health/publications/noise2.pdf. Acesso em Acesso em $17 \mathrm{de}$ fevereiro de 2019.

ANDERSON, K.; MATKIN, N. Relationship of Hearing Loss to Listening and Learning Needs: Educational Audiology Association Listserv. 2007.

CONAFE. Discapacidad auditiva - Guía didáctica para la inclusión en educación inicial y básica: Consejo Nacional de Fomento Educativo. Gobierno Federal del México. 2010.

DAVIS, A. et al. Children with Mild and Unilateral Hearing Impairment. Proceedings of the Second International Conference: A Sound Foundation Through Early Amplification.Section V. Chapter 14. 2002

KADERAVEK, J.N.; PAKULSKI, L.A. Minimal Hearing Loss Is Not Minimal. Teaching Exceptional Children. Council for Exceptional Children. v. 34, n.6, p.14-18. 2002.

MCGRATH, M. Auditory System Image. Disponívem em: drmarkmcgrath com.au. Acesso em 17 de fevereiro de 2019.

New Zeland Governament. What is mild hearing loss?: Ministry of Health of New Zeland 2014. Disponível em: https://www.nsu.govt.nz/system/files/resources/mild-hearing-loss.pdf, Acesso em: Acesso em 17 de fevereiro de 2019.

$$
\text { RELEDUC | ISE | v. } 2 \text { | n. } 1 \text { | fev. } 2019
$$


SINAUER ASSOCIATES. Neural System Image. Disponível em: https://neuroscience5e.sinauer.com/index.html. Acesso em 17 de fevereiro e 2019.

THARPE, A.M.; BESS, F. Identification and management of children with minimal hearing loss. International Journal of Pediatric Otorhinolatyngology. v.21, n.1, p.41-50, 1991.

Minimal, Progressive, and Fluctuating Hearing Losses In Children: Characteristics, Identification, and Management. Pediatric Clinics of North America. v.46, n.1, p. 65-78I. 1999.

THARPE, A.M. Minimal Hearing Loss in Children: The Facts and the Fiction. A Sound Foundation Through Early Amplification: Phonak, 2007.

Unilateral and Mild Bilateral Hearing Loss in Children: Past and Current Perspectives. Trends in Amplification. v. 12, n. 1, p.7-15. 2008.

WAKE, M.; POULAKIS, Z.J. Slight and mild hearing loss in primary school children. Paediatr. Child Health. v.40, n.1, p. 11-13. 2004.

WERFEL, K.L.; HENDRICKS, A.E. Identifying Minimal Hearing Loss and Managing Its Effects on Literacy Learning. TEACHING Exceptional Children. vol. 48, n. 4, pp. 213-217. 2015.

WHO. Hearing loss due to recreational exposure to loud sounds: World Health Organization. Ocupational health. 2015. Disponível em: https://apps.who.int/iris/bitstream/handle/10665/154589/9789241508513_eng.pdf;jsessionid= 513BF228E093F4BDD5CEBE9DB7E1A620?sequence=1. Acesso em 17 de fevereiro de 2019

YOSHINAGA-ITANO, C. et al. Outcomes of Children with Mild Bilateral Hearing Loss and Unilateral Hearing Loss. Seminars in Hearing. v. 29, n.2. p. 196-211. 2008.

ZAHNERT, T. The Differential Diagnosis of Hearing Loss. Dtsch Arztebl Int. v. 108, n.25, p. 433-444. 2011. 